\title{
Studying the Preheating Effect on Certain Mechanical Properties of the Coating Layer by Vacuum Ion Plasma Coating
}

\author{
Abdul Jaleel Hamad Majeed \\ Industrial Engineering Department, University of Jordan, Amman, Jordan \\ Email: almajeed.jaleel@gmail.com
}

Received 4 August 2015; accepted 6 November 2015; published 11 November 2015

Copyright (C) 2015 by author and Scientific Research Publishing Inc.

This work is licensed under the Creative Commons Attribution International License (CC BY). http://creativecommons.org/licenses/by/4.0/

c) (i) Open Access

\section{Abstract}

The present paper is concerned with the study of two main points: clarifying the effect of preheating on the surface microscopic shape of coating layer by vacuum ion plasma coating technology and on the amount of micro-hardness besides studying the properties of the surfaces that concern the parts of the mechanical system because of their direct and active effect on the machine efficient performance and machine life in general and their important effect on the product efficiency. As known, the instruments that measure the traditional roughness give us numbers and charts that, to a certain extent, describe the actual state of the surface shape within limited range of accuracy, but it has become necessary to find more accurate methods that suit the development obtained in industry, namely, the important applications like aircraft missiles and others. One of the suggested solutions for this is to get benefit of average fractal dimension for the surface by the help of the new technologies which, in return, help in the possibility to determine the surface state with high accuracy that matches the importance of application.

\section{Keywords}

Fractal Dimension, Roughness, Preheating, Ion-Plasma, Surface Homogeneity

\section{Introduction}

Modern engineering problems not only pose challenges in the search for new materials processing, but also require a search for new ways to evaluate their properties, especially in the field of micro-industries and processors surfaces using nanotechnology as well as physical and chemical methods. The challenge of modern machining industries is mainly focused on the achievement of high quality, in terms of work piece dimensional ac- 
curacy, surface roughness, high production rate, less tool wear on the cutting tools, economy of machining in terms of cost saving and increase the performance of the product [1].

The industry and the market require products to possess the best utilizable properties possible. The setting up of higher and higher demands is caused by the development of motor-car industry, air-industry, space-industry, precision engineering and other advanced technologies [2].

Recently, the traditional methods study the case of measuring the surface roughness by: maximum height roughness and surface roughness average score, etc. [3]. But as noted by some researchers [4], despite the success of these methods, there is still an urgent need to search for new solutions to evaluate the actual micro-relief properties of surfaces that display not only its amplitude characteristics, but also its structure.

One of the solutions may adopt advanced methods of dealing in determining the properties of surfaces using the basic principles of the geometric pattern repeated images theory which is mentioned in a number of scientific articles [5] [6]. This provides new horizons of the techniques presented to evaluate the surface layer quality of machine parts based on the fractal dimension of the surface.

Applicability of fractal geometry in describing some grossly irregular and fragmented faces of nature is so strikingly evident from the illustrations that it is reasonable to wonder why it had not been heard of before 1975 .

The first important parameter is angle of lighting and of observation. The second parameter, denoted $D$, is the most interesting and the most important one. In the case of surfaces, it lies between two and three [7].

We shall consider $R$ synthetic or natural surfaces under increasing magnifications. The surfaces are required in such a way that the domain of definition of the $r$ th surface is

$$
\left[-\frac{a}{2^{r}}, \frac{a}{2^{r}}\right] \times\left[-\frac{b}{2^{r}}, \frac{b}{2^{r}}\right], r=1, \cdots, R,
$$

where $a$ and $b$ are width and height of the surface under the lowest magnification, that is to say, magnification sequentially doubles. The surfaces are discredited into a finite number of sample points. The points in each surface are equally spaced, both in $x$ and $y$ direction, see Figure 1. The number of points along $x$ and $y$ axes in each surface is $N$ and $M$, respectively. We shall denote independent coordinate of $i j$ th sample point of $r$ th surface as

$$
z_{i j}^{(r)}, i=1, \cdots, N, j=1, \cdots, M, r=1, \cdots, R .
$$

The $r$ th surface is then approximated by the set $z_{i j}^{(r)}$, which will be referred to as $r$ th data set.

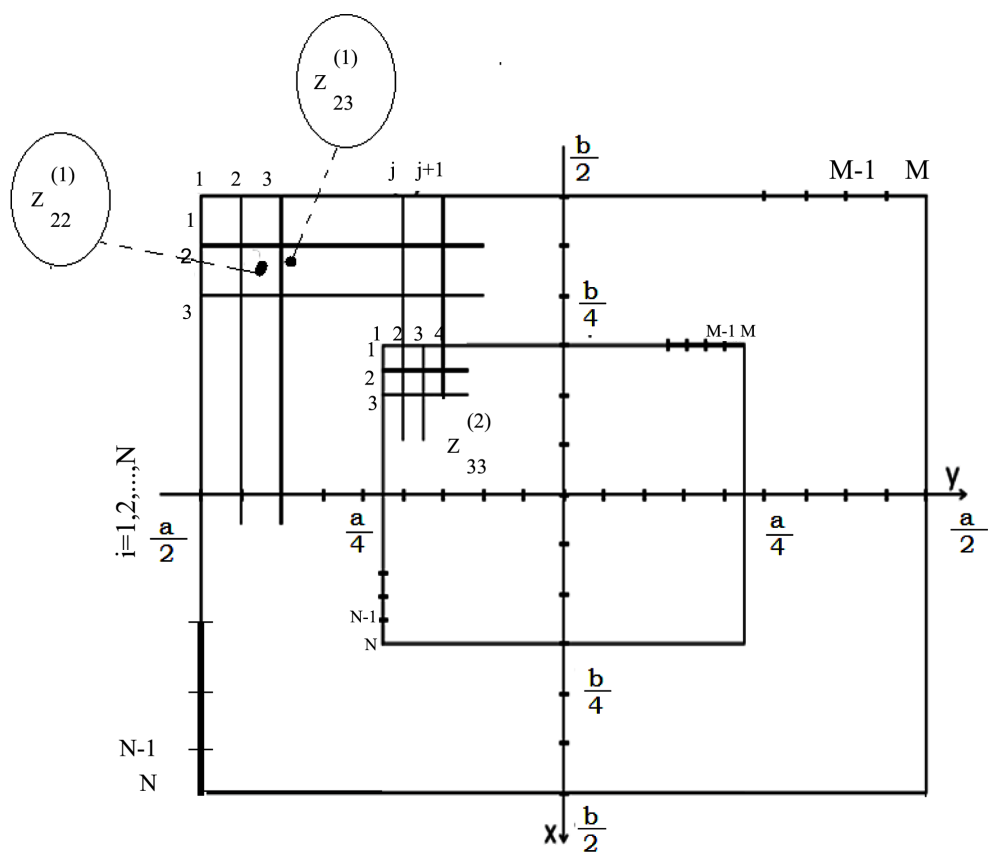

Figure 1. Notation for discretization of the first two data sets [4]. 
In the experiment, the original stereo pairs were assumed to be concentric, but in reality the photos with successive magnifications could be shifted by up to 10 pixels. This slight eccentricity should not affect the results [4].

The purpose of this paper is to study and evaluate the preheating effect on the micro-hardness value of the surface, as well as to know the exact surfaces properties by determining the average value of the fractal dimension.

The purpose of this paper is to study and evaluate the preheating effect on the micro-hardness value of the surface, as well as to know the exact surfaces properties by determining the average value of the fractal dimension.

\section{Experimental Work and Results}

First, the measuring of surface roughness by traditional methods is based mainly on the diamond tip radius, which are sensitive to the surface nature, and transfer oscillation motion on the surface to specific values that give a clear view of the surface state, and this recognizes the importance specifications of the end of the probe and its impact increased efficiency fumbling, diamond tip radius $5 \mu \mathrm{m}$. This means that traditional methods of measuring surface roughness do not give the required full description for all surfaces, especially surfaces that are used in very important applications. Therefore, we must benefit from the fractal dimension property of the surface that gives most accurate description, especially its benefit from the magnification power of microscope and then analyze the surface taken image and find the average fractal dimension by using special software for calculating. The analysis of the picture of the surface obtained as a bit-map, is processed by the Fractal programme 3.4.7.2009, and its supporting applications [8].

The used sample has coefficient of surface roughness $\mathrm{Ra}=0.647 \mu \mathrm{m}$, by calculating the average fractal dimension for different magnification power of image as shown in Figure 2, significantly observed. By taking all this into account the investigations [9] [10].

Second, we studied the temperature effect on the coated piece by using vacuum ion-plasma coating technology, and preheating effect on the surface nature and mechanical properties. We had a test on three groups of specimens of high speed steel R6 M5, all equal in dimensions (diameter of $12 \mathrm{~mm}$ and a thickness of $4 \mathrm{~mm}$ ).

We measured the average surface roughness factor $(\mathrm{Ra})$ for each specimen five times and calculated the average value. Surface roughness plays a dominant role in increasing the oxidation rate of metals and alloys [11] [12]. Coating by vacuum ion plasma was used to improve most of the mechanical properties. Then we measured the micro-hardness before and after coating processes that took place in three different temperatures while retaining other coating conditions like, pressure, time, etc., which were equal. These steps were done for the purpose of determining the preheating effect on the coating specifications. The surface roughness for specimens

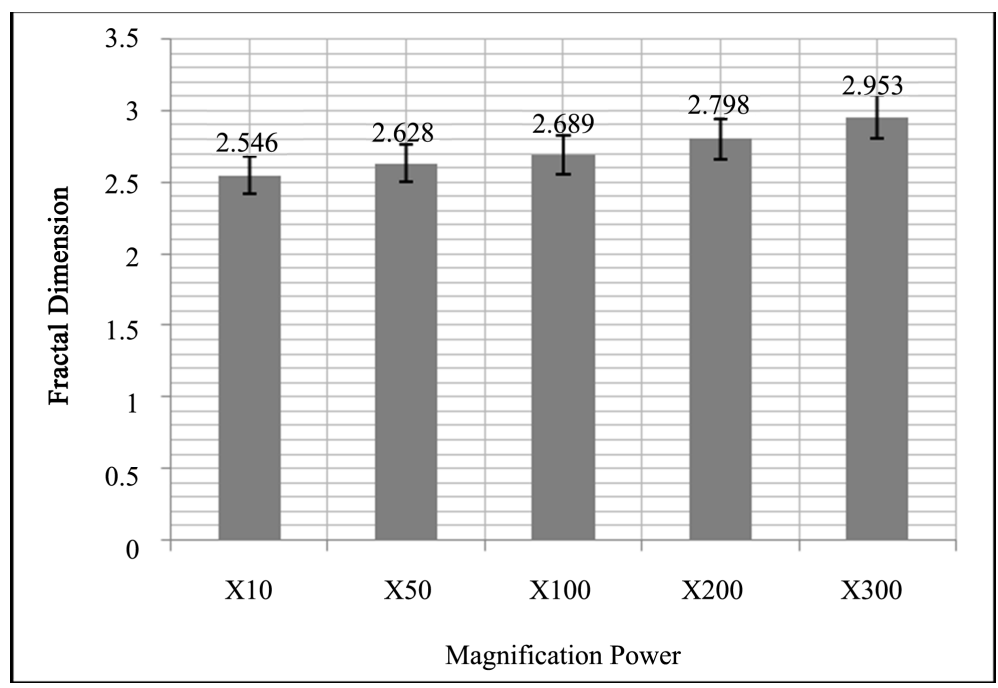

Figure 2. Shows the effect of magnification power on the amount of fractal dimension, of specimen $(\mathrm{Ra}=0.647 \mu \mathrm{m})$. 
(Group 1) before coating is $0.471 \mu \mathrm{m}$, and the other details are shown in Table 1.

The surface roughness that Concern specimens (Group 2) before coating is $0.191 \mu \mathrm{m}$, and other details are shown in Table 2.

The surface roughness that Concern specimens (Group 3) before coating is $0.142 \mu \mathrm{m}$, and other details are shown in Table 3.

We use average amount of fractal dimension for each sample with the help of licensed software and submitted by the Japanese Research Center. Microscopic imaging processes were used an electronic microscope type Neophot 2 as shown in Figure 3 with different enlargement power as follows: X10, X50, X100, X200, X300 and calculated the average count Fractal for each power zoom, and measured Micro-hardness for each specimen before and after coating processes. A device used for measuring micro-hardness shown in Figure 4. For the coating processes, Coating machine model NN6.6-E1 was used, as shown in Figure 5. As shown in Figure 6 the results explained clearly the effect. The chemical composition of coating pole (cathode) is shown in Table 4 .

\section{Conclusions}

The main conclusions are summarized as follows:

1. Preheating temperature plays an important role in increasing the micro-hardness amount of the metal to be coated.

Table 1. The details that concern the first group specimens.

\begin{tabular}{|c|c|c|c|c|c|}
\hline \multicolumn{2}{|c|}{ Coating Temperature $\left({ }^{\circ} \mathrm{C}\right)=400-420$} & & & & \\
\hline Surface roughne & & \multicolumn{4}{|c|}{ Group 1} \\
\hline \multicolumn{2}{|c|}{ Micro-hardness = $15.19 \mathrm{GPa}$} & & & & \\
\hline Magnification Power & $\mathrm{X} 10$ & X50 & $\mathrm{X} 100$ & X200 & X300 \\
\hline Fractal Dimension (FD1) & 2.552 & 2.628 & 2.689 & 2.798 & 2.953 \\
\hline
\end{tabular}

Table 2. The details that concern the second group specimens.

\begin{tabular}{|c|c|c|c|c|c|}
\hline \multicolumn{2}{|c|}{ Coating Temperature $\left({ }^{\circ} \mathrm{C}\right)=500-520$} & & & & \\
\hline Surface roughn & & \multicolumn{4}{|c|}{ Group 2} \\
\hline \multicolumn{2}{|c|}{ Micro-hardness = $19.37 \mathrm{GPa}$} & & & & \\
\hline Magnification Power & $\mathrm{X} 10$ & X50 & $\mathrm{X} 100$ & X200 & X300 \\
\hline Fractal Dimension (FD1) & 2.546 & 2.533 & 2.527 & 2.526 & 2.511 \\
\hline
\end{tabular}

Table 3. The details that concern the third group specimens.

\begin{tabular}{|c|c|c|c|c|c|}
\hline \multicolumn{2}{|c|}{ Coating Temperature $\left({ }^{\circ} \mathrm{C}\right)=600-620$} & & & & \\
\hline Surface roughn & & \multicolumn{4}{|c|}{ Group 3} \\
\hline \multicolumn{2}{|c|}{ Micro-hardness $=18.36 \mathrm{GPa}$} & & & & \\
\hline Magnification Power & $\mathrm{X} 10$ & $\mathrm{X} 50$ & $\mathrm{X} 100$ & X200 & X300 \\
\hline Fractal Dimension (FD1) & 2.549 & 2.577 & 2.698 & 2.867 & 2.894 \\
\hline
\end{tabular}

Table 4. Cathode chemical composition.

\begin{tabular}{ccccccccc}
\hline & \multicolumn{7}{c}{ (Cathode) Chemical Composition \% Mass } \\
\hline $\mathrm{Ti}$ & $\mathrm{C}$ & $\mathrm{O}_{2}$ & $\mathrm{~N}$ & $\mathrm{H}$ & $\mathrm{Al}$ & $\mathrm{Si}$ & $\mathrm{Fe}$ & Others \\
\hline Base & 0.06 & 0.18 & 0.04 & 0.005 & 0.41 & 0.03 & 0.18 & 0.30 \\
\hline
\end{tabular}




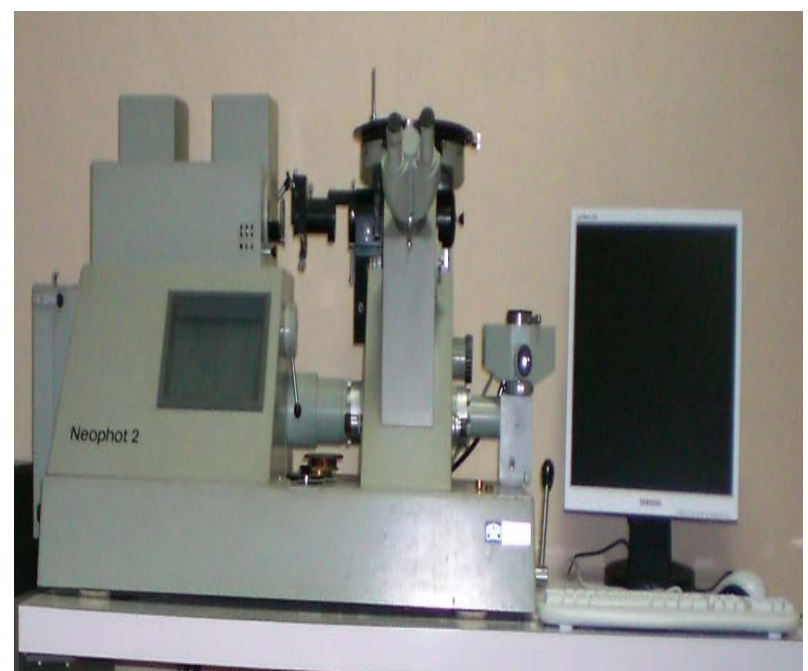

Figure 3. Electronic microscope model Neophot 2.

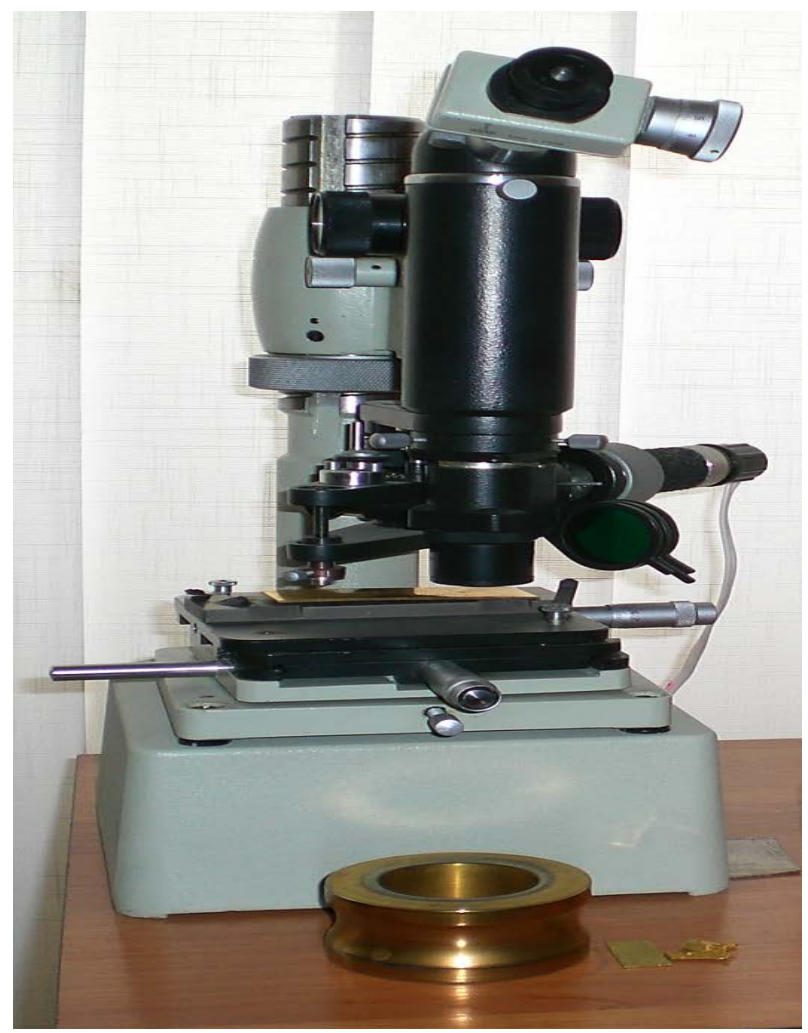

Figure 4. For measuring ricro hardness model PMT-3.

2. Preheating temperature interferes with direct intervention and the consistency of the outer layer of the surface.

3. The results obtained allow us to evaluate the effect of different treatments of surfaces by identification the fractal dimension.

4. The average roughness factor $(\mathrm{Ra})$ does not necessarily mean the consistency of the outer coated layer of the surface.

5. The possibility of the follow-up state of the surface is good besides much higher resolution than traditional methods, which helps to obtain the required specifications. 


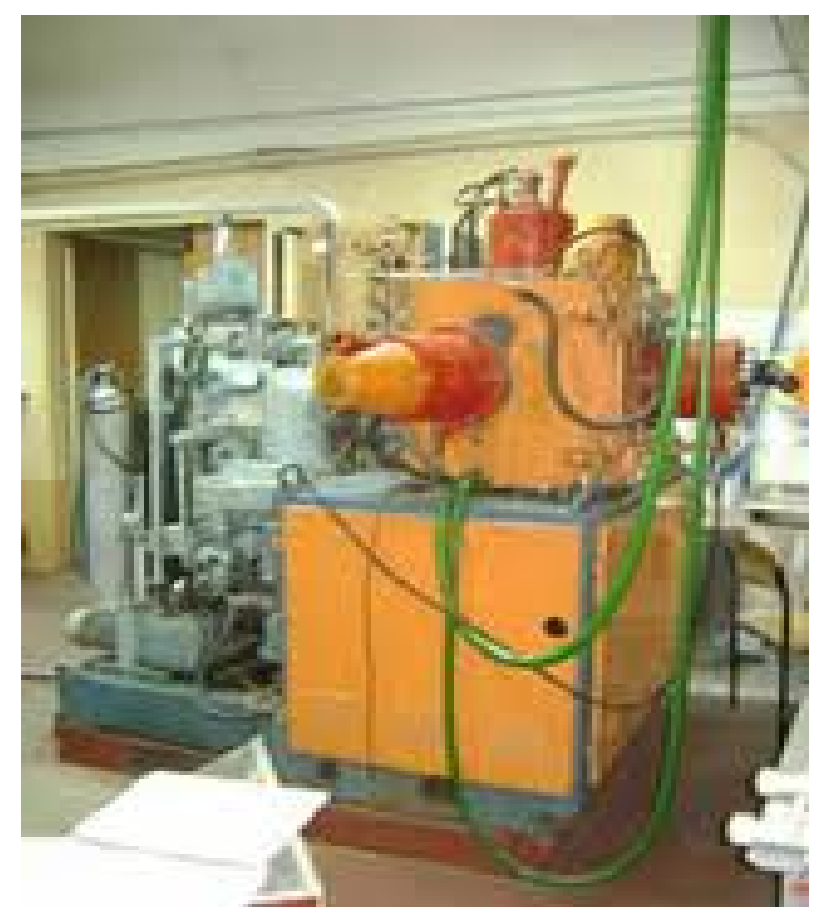

Figure 5. Coating Machine Model NN6.6-E1.

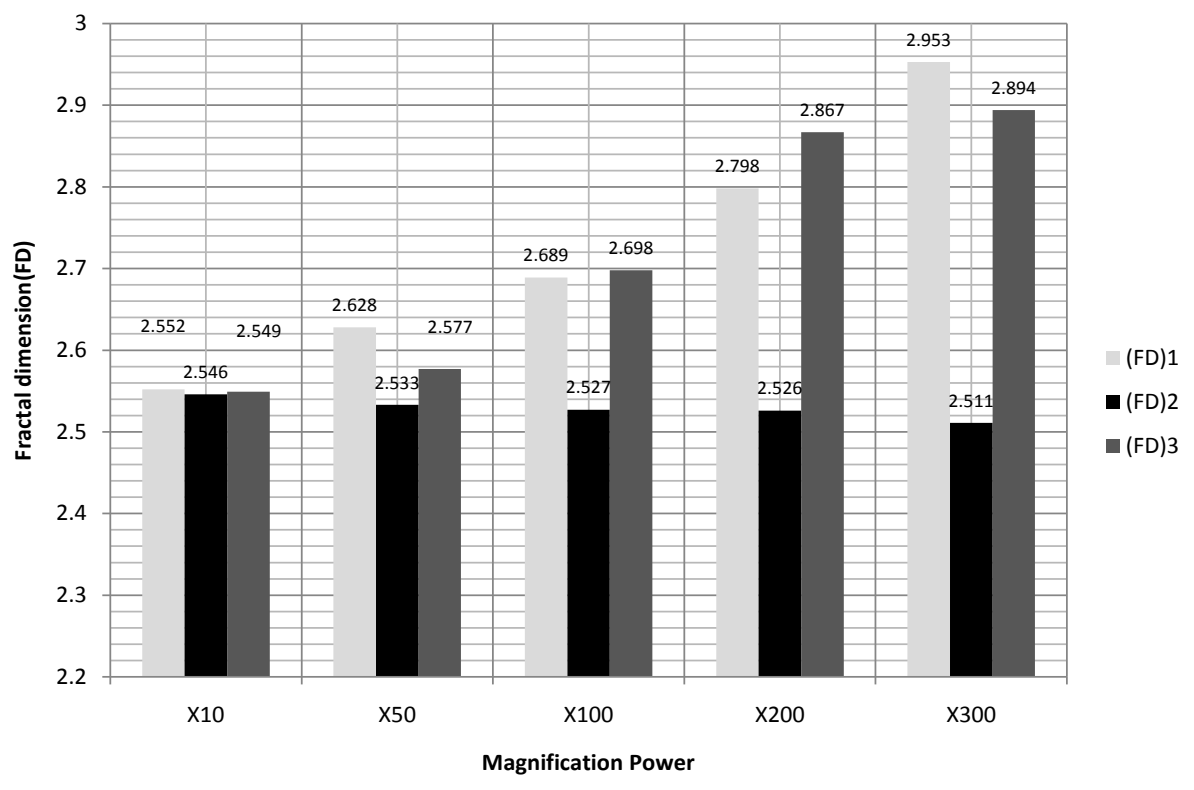

Figure 6. The effect of preheating on the surface shape by using average fractal dimension. FD1: average fractal dimension for the specimens (Group 1) coated at temperature $400^{\circ} \mathrm{C}$ $420^{\circ} \mathrm{C}$. FD2: average fractal dimension for the specimens (Group 2) coated at temperature $500^{\circ} \mathrm{C}-520^{\circ} \mathrm{C}$. FD3: average fractal dimension for the specimens (Group 3) coated at temperature $600^{\circ} \mathrm{C}-620^{\circ} \mathrm{C}$.

6. We can know the amount of the surface affected by the finishing processes, whether mechanical operations, traditional or non-traditional.

7. Fractal dimension describes precisely the effect nature on the ductile and brittle surfaces with various mechanical stresses, thermal and chemical.

8. Fractal dimension provides depth and comprehensive knowledge when studying surfaces by it. 


\section{References}

[1] Thamizhmanii, S., Bin Omar, B., Saparudin, S. and Hasan, S. (2008) Surface Roughness Analyses on Hard Martens Tic Stainless Steel by Turning. Journal of Achievements in Materials and Manufacturing Engineering, 26, 139-142.

[2] Legutko, S., Kluk, P. and Stoic, A. (2011) Research of the Surface Roughness Created during Pull Broaching Process. METABK, 50, 245-248.

[3] Buj-Corrral, I. and Vivancos-Calvet, J. (2011) Roughness Variability in the Honing Process of Steel Cylinder with CBN Metal Bonded Tools. Precision Engineering, 35, 289-293. http://dx.doi.org/10.1016/j.precisioneng.2010.11.004

[4] Ruzicka, S. and Hausild, P. (2010) Fractal Aspects of Ductile and Cleavage Fracture Surfaces. International Journal of Machine Tools and Manufacture, 77, 744-752.

[5] Feder, J. (1988) Fractals. Plenum, New York.

[6] Bojokin, S.V. and Parshin, D.A. (2001) Fractals and Multifractals. Regular and Chaotic Dynamics, NITs, Izhevsk, 128 p.

[7] Mandelbrot, B.B. (1982) Fractal Geometry of Nature. Freeman, San Francisco.

[8] Fraczek, J., Kaczorowski, J. and OElipek, Z. (2000) Measurement of Grain Surface Roughness. International Agrophysics, 14, 167-172.

[9] Leonid, S., Valeriy, G. and Boris, B. (2012) Investigation of Porosity and Fractal Properties of the Sintered Metal and Semiconductor Layers in the MDS Capacitor Structure. Science of Sintering, 44, 95-101. http://dx.doi.org/10.2298/SOS1201095S

[10] Sanchez-Molina, D., Velazquez-Ameijide, J., Quintana, V., Arregui-Dalmases, C., Crandall, J.R., Subit, D. and Kerrigan, J.R. (2013) Fractal Dimension and Mechanical Properties of Human Cortical Bone. International Journal of Machine Tools and Manufacture, 35, 576-582.

[11] Edgar, G. (2000) Measure, Topology, and Fractal Geometry. 2nd Edition, Springer, USA.

[12] Roy, M., Ray, K.K. and Sundararajan, G. (1999) The Influence of Erosion-Induced Roughness on the Oxidation Kinetics of $\mathrm{Ni}$ and $\mathrm{Ni} \pm$ 20Cr Alloys. Oxidation of Metals, 51, 251-272.

\section{Nomenclatur}

$\begin{array}{ll}F D & \begin{array}{l}\text { fractal dimension } \\ R a\end{array} \\ a \times b & \text { roughness }(\mu \mathrm{m}) \\ R & \text { dimensions of the largest photograph } \\ N \times M & \text { number of concentric photographs } \\ z_{i j}^{(r)} & \text { number of points of the reconstructed surface } \\ Y & \text { vertical coordinate of } i j \text { th reconstructed point } \\ & \text { number of dividers }\end{array}$

